\section{Seroprevalence of Cytomega- lovirus among Healthy Blood Donors in Sana'a City, Yemen}

\author{
Ammar MA AI-Sabri ${ }^{1,3}$, Saad Al-Arnoot ${ }^{2,4 *}$, Anwar K Al-Madha- \\ gi $^{1}$ and Hassan A Al-Shamahy ${ }^{1}$
}

${ }^{1}$ Department of Medical Microbiology, Faculty of Medicine and Health Sciences, Sana'a University, Sana'a, Yemen

${ }^{2}$ Microbiology Division, Department of Biology, Faculty of Science, Sana'a University, Sana'a, Yemen

${ }^{3}$ Virolology Department, National Blood Transfusion and Research Center, Sana'a, Yemen

${ }^{4}$ Microbiology Department, Quality Control lab, Yemen Egyptian Pharma Co. Itd, Sana'a, Yemen

\begin{abstract}
Cytomegalovirus (CMV) is a member of the human herpes family of viruses. It has emerged as a significant cause of morbidity and mortality in children and immunocompromised adults. A cross-sectional study was conducted to determine CMV prevalence among male healthy blood donors attending National Blood Transfusion and Research Center in Sana'a city, Yemen. Serum samples were tested for CMV-specific immunoglobulin $G$ and immunoglobulin $M$ from the participants by Enzyme Linked Immunosorbent Assay (ELISA). Out of 235 subjects, 227(96.6\%) were seropositive for CMV IgG while $13(5.5 \%)$ were seropositive for CMV IgM. There was no statistically significant associated with CMV seropositive and age group $(P>0.05)$. This study revealed the high percentage of CMV seropositive blood donors indicates that CMV infection is common in our population. More studies are needed to elucidate the transmission of transfusion-associated CMV before establish proper guidelines on routine CMV screening in blood donors.

Keywords: Blood donors; Cytomegalovirus; ELISA; Seroprevalence; Sana'a; Yemen
\end{abstract}

\section{Introduction}

Cytomegalovirus (CMV) is a member of the human herpes family of viruses. It has emerged as a significant cause of morbidity and mortality in children and immunocompromised adults. It transmitted by direct or indirect, close or intimate person-to-person contact. Sources of the virus include oropharyngeal secretions, urine, cervical, vaginal excretions, semen, breast milk, tears, faeces and blood [1].

*Corresponding author: Saad Al-Arnoot, Microbiology Division, Department of Biology, Faculty of Science, Sana'a University, Sana'a, Yemen, Tel: +967 00967774667900; E-mail: saad.alarnoot@gmail.com

Citation: Al-Sabri AMA, Al-Arnoot S, Al-Madhagi AK, Al-Shamahy HA (2017) Seroprevalence of Cytomegalovirus among Healthy Blood Donors in Sana'a City, Yemen. J Infect Non Infect Dis 3: 023.

Received: March 03, 2017; Accepted: April 06, 2017; Published: April 20, 2017
According to the result of a WHO cooperative study involving equal numbers of male and female healthy blood donors between 20-40 years of age are shown. The seroprevalence of antibody in that study ranges from $40-100 \%$ [2]. However, in Yemen, a few studies have investigated the prevalence of CMV antibody which seroprevalence of CMV antibody ranges between $68 \%-100 \%$ among pregnant women [3-5].

The American Association of Blood Banks has recommended transfusion from donors who are seronegative for CMV, or the use of deglycerolised Frozen Red Blood Cells (RBCs) for transfusion in a seronegative preterm $(<1200 \mathrm{~g})$ child born to a mother with negative or unknown immune status regarding CMV infection [6]. These guidelines have helped to eliminate transfusion-induced CMV infection syndrome in preterm infants in the West. However, since the vast majority of blood donors in developing countries are seropositive for $\mathrm{CMV}$, it would be imprudent to screen blood donors for CMV as very few seronegative blood units would be available for transfusion. Other preventive strategies (such as leukoreduction filtration, saline-washed RBCs, frozen deglycerolised RBCs, etc.,) are increasingly recommended to minimize transfusion transmission of CMV. These methods may be more appropriate and cost effective in developing countries for the prevention of CMV transmission through infected blood to immunosuppressed individuals. More studies are needed to elucidate the transmission of transfusion-associated CMV before proper guidelines on routine CMV screening in blood donors can be formulated [7].

Like other developing countries Yemeni blood donors are screened only for HIV, Hepatitis B Virus (HBV), Hepatitis C Virus (HCV) and Syphilis. Also, there is no published data concerning CMV seroprevalence in blood donors in Yemen. Therefore the present study was undertaken to determine the prevalence of antibodies to CMV infection among male blood donors at the National Blood Transfusion and Research Center in Sana'a city, Yemen. It's a useful study for understanding situation of CMV infection in Yemen and offering the helpful data for government policymakers.

\section{Materials and Methods}

A cross-sectional study was collected from 235 male healthy voluntary donors attending National Blood Transfusion and Research Center in Sana'a city, Yemen. About $5 \mathrm{ml}$ blood samples was collected from each consenting subjects by venipuncture, transferred into sterile anticoagulant-free sterile bottle and allowed to clot. The clotted blood sample was centrifuged ( $3000 \mathrm{rpm}, 5 \mathrm{~min}$ ) and the serum was transferred into cryovials and stored at -10 to $-20^{\circ} \mathrm{C}$ until required for use. The samples were analyzed for CMV-specific Immunoglobulin (Ig) IgG and IgM by Enzyme Linked Immunosorbent Assay (ELISA) test kits (Calbiotech INC (CBI) Life Science Company, USA) in accordance with the manufacturer's instructions. Positive and negative standard sera, accompanying the kit were included in each assay. The ethical clearance for this research was granted by the Department of Medical Microbiology, Faculty of Medicine and Health Sciences, Sana'a University ethical committee after due process had been followed. Statistical analysis of results was done using the R software. Fisher exact test and a $\mathrm{p}$ value of $<0.05$ was considered significant. 
Citation: Al-Sabri AMA, Al-Arnoot S, Al-Madhagi AK, Al-Shamahy HA (2017) Seroprevalence of Cytomegalovirus among Healthy Blood Donors in Sana'a City, Yemen. J Infect Non Infect Dis 3: 023.

- Page 2 of $3 \cdot$

\begin{tabular}{|c|c|c|c|c|}
\hline Age groups (Years) & Participants No (\%) & IgG Positive No (\%) & P-value & IgM Positive No (\%) \\
\hline $20-24$ & $70(29.79)$ & $67(95.71)$ & 0.4009 & $4(5.71)$ \\
\hline $25-29$ & $54(22.98)$ & $50(92.59)$ & $1(1.85)$ \\
\hline $30-34$ & $64(27.23)$ & $63(98.44)$ & $5(7.81)$ \\
\hline $35-39$ & $20(8.51)$ & $20(100)$ & $2(10.0)$ \\
\hline$\geq 40$ & $27(11.49)$ & $27(100)$ & $1(3.70)$ \\
\hline \multicolumn{2}{|c|}{ Table 1: Distributions of CMV IgG and IgM antibodies among healthy male voluntary donors according to their age. } \\
\hline
\end{tabular}

\section{Results and Discussion}

To our knowledge, this is the first report on the seroprevalence of CMV antibodies among male healthy blood donors in Yemen. Their age ranged from 20 to 48 years with mean age of 29.1 years. Out of 235 subjects, 227(96.6 \%) were seropositive for CMV IgG indicative of exposure to CMV while only 13(5.5\%) were seropositive for CMV IgM. The age wise distribution of CMV IgG and CMV IgM seropositive donors is given in table 1 . The results of this study showed the prevalence rate of CMV IgG antibodies $(96.6 \%)$ were similar with previous studies performed the country in Sana (100\%) and Hodeidah $(98.7 \%)$ among pregnant women $[3,4]$. The results from our study nearly similar with studies performed in other developing countries in Iraq (97.4 \%) and Iran (99.2\%) among blood donors [8,9]. This rate $96.6 \%$ is higher than that reported by other studies performed in Japan and India showed $84 \%$ and $61 \%$ of adults had the antibodies respectively $[10,11]$. On the other hand, WHO sponsored survey of complement-fixing antibodies against CMV and reported frequencies ranging from $40 \%$ in highly industrialized areas to $100 \%$ in developing countries of CMV-IgG antibodies [2,12].

The seropositive for CMV IgG antibodies among Yemeni blood donors was $96.6 \%$ indicating past exposure to infection the high seroprevalence in Yemen and developing countries as India, Brazil and Nigeria is in contrast to Western literature which describes seroprevalence IgG in voluntary blood donors ranging from $38 \%$ to $75 \%$ in different parts of the developed world [13-15]. The high seroprevalence among Yemeni populations indicates the endemicity of infection. This could be related to the low socioeconomic status in Yemen which reflects increased exposure to CMV due to factors including large household size, crowding, certain child care practices and possibly sexual practices $[11,12,16]$. Although several studies on the prevalence of CMV antibody in different parts of the world have been done, the results were not comparable in detail because methodologies used are different. Interestingly, the prevalence of antibody in Japan and Hong Kong is over 90\%, although they cannot be considered 'developing' nations [11].

In this study, the seroprevalence of CMV IgG among the blood donors varied with age ranging from $92.6 \%$ in the $25-29$ year age group to $100 \%$ in the $>40$-year age group (Table 1 ). The decrease in the percentage seropositivity in the 25-29 year age group is most likely due to the fact that data in this age band were based on smaller numbers.

The result from this current study showed 5.5\% (13/235) of the blood donors tested were positive for CMV IgM indicating the presence of primary recent CMV infection among them. This IgM rate is lower than that reported from Nigeria in which $19.5 \%$ of healthy blood donors had CMV IgM antibodies while this rate is higher than that reported by other studies performed in Iraq and Iran showed $1.6 \%$ and $1.0 \%$ of healthy blood donors had CMV IgM antibodies respectively $[8,13,17]$. While this rate is lower than that reported by other studies performed in Nigeria 28.0\% [18].
There is no statistically significant difference in seropositivity of CMV based on distribution of age. This differs from previous studies, in which this antibody was more prevalent in older age ranges Brazil studies which showed a significantly increased seropositivity with increasing age of blood donors [14]. This may possibly due to earlier acquisition of CMV infection in Yemen in childhood compared to the western populations, leading to higher seroprevalence even in younger adults $[10,19]$.

Limitations of the study included the relatively small sample size (which is due to financial constraints in acquiring enough test kits). The other limitation we did not use Polymerase Chain Reaction (PCR) of the viral DNA isolation due to financial constraints too.

\section{Conclusion}

This study revealed the high percentage (96.6\%) of CMV seropositive blood donors indicates that CMV infection is common in our population. More studies are needed to elucidate the transmission of transfusion associated CMV before establish proper guidelines on routine CMV screening in blood donors.

\section{Acknowledgement}

The authors were grateful to Department of Medical Microbiology, Faculty of Medicine and Health Sciences, Sana'a University, Sana'a, Yemen for supporting this research. We are also grateful to the National Blood Transfusion and Research Center for their cooperation.

\section{References}

1. Pass R (2001) Cytomegalovirus. In: Knipe DM, Howley PM, Griffin DE (eds.). Fields Virology (Vol2), Lippincott Williams \& Wilkins, Boston, USA. Pg no 2675-2705.

2. Krech $U$ (1973) Complement-fixing antibodies against cytomegalovirus in different parts of the world. Bull World Health Organ 49: 103-106.

3. Al-Samawi MMA (2003) Prevalence of Human Cytomegalovirus in Yemen. University of Jordan, Amman, Jordan.

4. Alghalibi SMS, Abdullah QYM, Al-Arnoot S, Al-Thobhani A (2016) Seroprevalence of Cytomegalovirus among Pregnant Women in Hodeidah city, Yemen. J Hum Virol Retrovirol 3: 00106.

5. Edrees A (2010) Prevalence Cytomegalovirus antibodies among pregnant women and newborns in the hospital president in Jebla, Ibb Yemen, Sana'a.

6. Roback JD (2002) CMV and blood transfusions. Rev Med Virol 12: 211-219.

7. American Association of Blood Banks (1987) Standards for blood banks and transfusion services (12thedn). American Association of Blood Banks, Committee on Standards, The Committee, Arlington, USA, Pg no: 30-31.

8. Khudir MK, Molan A-L (2014) Seroprevalence of cytomegalovirus among healthy students at Diyala University, Diyala, Iraq. Journal of Pharmacy and Biological Sciences 9: 59-61.

9. Safabakhsh H, Karimi G, Tehranian F, Tehranian B (2014) Demography and seroprevalence of cytomegalovirus infection in blood donors in Mashhad in 2009. J Am Sci 10: 139-142. 
Citation: Al-Sabri AMA, Al-Arnoot S, Al-Madhagi AK, Al-Shamahy HA (2017) Seroprevalence of Cytomegalovirus among Healthy Blood Donors in Sana'a City, Yemen. J Infect Non Infect Dis 3: 023.

10. Al-Jiffri O, Al-Sharif FM, El-Sayed ZM (2013) Seroprevalence of Cytomegalovirus among Blood Donors and Other Investigated Groups. Inter J Microbiol Res 4: 1-8.

11. Furui Y, Satake M, Hoshi Y, Uchida S, Suzuki K, et al. (2013) Cytomegalovirus (CMV) seroprevalence in Japanese blood donors and high detection frequency of CMV DNA in elderly donors. Transfusion 53: 2190-2197.

12. Cannon MJ, Schmid DS, Hyde TB (2010) Review of cytomegalovirus seroprevalence and demographic characteristics associated with infection. Rev Med Virol 20: 202-213.

13. Akinbami AA, Akanmu AS, Adeyemo TA, Wright KA, Dada MO, et al. (2009) Cytomegalovirus antibodies amongst healthy blood donors at Lagos University Teaching Hospital (Luth). S Afr Med J 99: 528.

14. Souza MA, Passos AM, Treitinger A, Spada C (2010) Seroprevalence of cytomegalovirus antibodies in blood donors in southern, Brazil. Rev Soc Bras Med Trop 43: 359-361.
15. Ziemann M, Krueger S, Maier AB, Unmack A, Goerg S, et al. (2007) High prevalence of cytomegalovirus DNA in plasma samples of blood donors in connection with seroconversion. Transfusion 47: 1972-1983.

16. Fox-Canale AM, Hope TJ, Martinson J, Lurain JR, Rademaker AW, et al. (2007) Human cytomegalovirus and human immunodeficiency virus type-1 co-infection in human cervical tissue. Virology 369: 55-68.

17. Moniri R, Mosayebii Z, Mossavi GA (2004) Seroprevalence of cytomegalovirus, hepatitis $B$, hepatitis $C$ and human immunodeficiency virus antibodies among volunteer blood donors. Iranian Journal of Public Health 33: 38-42.

18. Oladipo EK, Akinpelu OO, Oladipo AA, Edowhorhu G (2014) Seroprevalence of Cytomegalovirus (CMV) among Blood Donors at Bowen University Teaching Hospital Ogbomoso. American Journal of Medical and Biological Research 2: 72-75.

19. Lehrer S, Green S, Ramanathan L, Rosenzweig K, Labombardi V (2012) No consistent relationship of glioblastoma incidence and cytomegalovirus seropositivity in whites, blacks, and Hispanics. Anticancer Res 32: 1113-1115. 